\title{
The use of radio-telemetry in the study of animal physiology
}

\author{
By J. Bligh and J. W. Heal, Agricultural Research Council, Institute of Animal \\ Physiology, Babraham, Cambridge
}

\section{Introduction}

The ultimate goal of physiologists is to understand the functions of living organisms in their natural environment, but the processes that constitute life are so numerous and complex that functions must be studied singly in the laboratory under artificially simplified conditions in which concurrent and interacting events are reduced to a minimum or can be ignored. When such observations made in isolation are brought together, how nearly does the resulting synthesis approach the total integrated processes of life under natural conditions? This is one of the abiding questions in biological research. One way to explore this problem is to develop techniques to study the functions of conscious, intact and unrestrained animals in their natural, or near-natural, environments, and to see how well such measurements correlate with those made in the buffered environment of the laboratory.

Basically there are two ways of achieving this: either the recording system can be placed on or in the animal, or a system of wireless transmission can be used to transmit information from an animal to a remotely located recorder system. Animalor man-borne miniature recording systems are outside the scope of this paper, but should not be overlooked as a convenient means of recording physiological data from unrestrained animals. So far they have found little application in animal studies, partly because of size and partly because they can function unattended for only short periods. However, these may be only temporary limitations which will be overcome.

Most records of physiological functions are made in the laboratory by electrically activated pens on moving paper. The physiological phenomenon, which may be a movement, a pressure or temperature change, or a chemical or bioelectrical event, must act on a suitable sensor and, except for bioelectrical events, the force acting on the sensor must be converted or transduced into an electrical signal and conveyed via an amplifier to a recorder system.

The primary component of such a system is always the sensor-transducer which generates an electrical impulse proportional to the variations in the physiological function; the terminal component is always the display or recorder, and the connection between the two is traditionally by wires. Since telemetry simply means measurement at a distance, such a system constitutes wired telemetry. The signal can, however, be transmitted by the wire-less means of radio waves, which is what is meant by radio-telemetry. The point we wish to make is that in the telemetering 
of physiological information, the only distinction between wired and wire-less systems is the presence or absence of connecting wires (Fig. I). The sensors-transducers and the recording systems have no distinguishing features. The main theme of any discussion of radio-telemetry in physiology concerns the radio system itself rather than the transducers and recorder systems. Yet many of the practical problems in the use of radio-telemetry stem from the transducers rather than the transmission system. For the most part these are common to any technique involving chronic implantation, but since they are responsible for much of the frustration of trying to use radio-telemetry they must be touched on briefly.

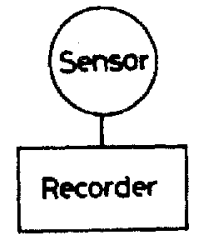

(a)

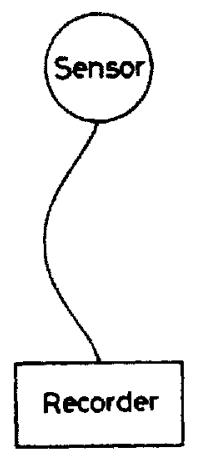

(b)

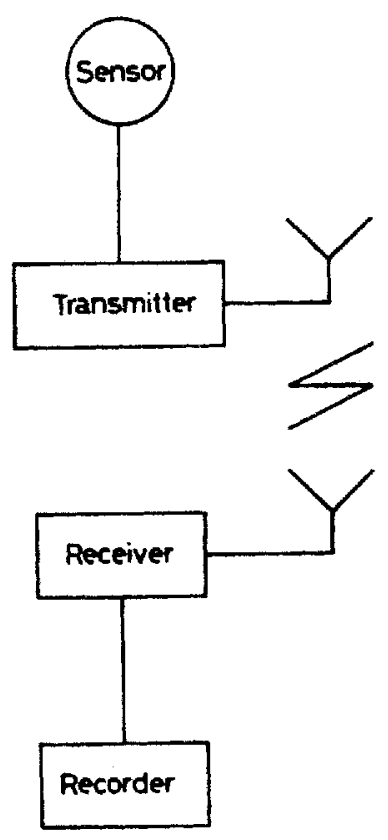

(c)

Fig. I. A schematic representation of the relations between sensor and recorder where: (a) the sensor and recorder are constructed as a single unit with a fixed and close relation between them; (b) the sensor is at a distance from the recorder, but connected to it by a flexible cable; and (c) there is no cable connection between sensor and recorder and information is transmitted from the one to the other by radio.

\section{Radio transmission systems}

Radio systems transmitting information about animals can be divided into two broad groups: (I) small, short-range devices which are embedded within the body and (2) larger devices which are held in harness on the body and, possibly, connected to implanted transducers.

\section{Implanted sensor-transmitter systems}

Where the transducer has to be implanted in the animal, it is preferable that the transmitter is also implanted because it eliminates the problem of wires passing 
through the skin. Transcutaneous connecting wires can be troublesome because they allow tracking infection by pathogenic organisms, and the continual flexing of the wires as the animal moves causes fracture sooner or later. However, the small embeddable transmitter also has its disadvantages. Body fluids eventually penetrate any container which is not totally encapsulated in an impermeable sheath, and such penetration by body fluids causes both short-circuiting and corrosion. The power pack must be encapsulated with the transmitter, and must therefore last for as long as the embedded transmitter is required to operate. The size of the power pack required is proportional to the intended duration of transmission, the range of detectable transmission, and the complexity of the circuit, but its physical dimensions and weight must be contained within limits. Thus a device suitable for transplantation must have a simple circuit and the signal is necessarily only low-powered. The internal aerial cannot be efficient and will almost certainly be sharply directional. This means that the implanted transmitter can be used for recording information from an unrestrained animal only if the animal is confined to a small area encircled by a receiver aerial.

The most effective of the implanted 'radio pills' has been that for sensing and transmitting thermal information. An oscillator running at several hundred $\mathrm{kHz}$ generates brief pulses at intervals of about $\mathrm{I} \mathrm{s}$. The exact frequency of the pulses is determined by the internal leakage current in the oscillating transistor, and this current is directly related to temperature. Thus in this instance the transmitter circuit is also the temperature transducer. An ordinary portable transistor radio can be used for listening to the transmitted 'clicks', but must be held within $0.5 \mathrm{~m}$ of the transmitter. Temperature is determined by counting pulses over a period of $\mathrm{I}$ min, and relating this frequency to the prior calibration made with the transmitter immersed in water at various temperatures. The decoding process can, of course, be automated and the temperature displayed or recorded in digital form.

Because the temperature 'pill' transmitter is its own transducer, and because it has a duty cycle of only about $r: 1000$, (i.e. it is transmitting for only $r / 1000$ th of the time) the whole device can be made small enough to be swallowed or implanted, and yet can have an operating life of many days. Other versions of the encapsulated 'pill' have been used for the measurement of pressure and $\mathrm{pH}$, but in these instances the transmission is continuous and the battery life is much reduced.

Where the implantation of rather larger devices is feasible (for example in the abdominal cavity), they can be used for a variety of purposes where continuous information or details of rapid changes are required, such as in the sensing and transmitting of changes in biological electrical potentials, or of changes in strain or movement.

\section{Harness-borne transmitters}

With larger external transmitters, signal-coded information on almost any physiological process can be transmitted over virtually any distance, as has been exemplified by the transmission to earth of physiological data from men walking on the moon. The practical limits imposed by consideration of cost gives results which are con33 (2) 7 
siderably less spectacular. In general, overland transmission from a feasibly-sized transmitter for animal studies may extend to several $\mathrm{km}$ in the absence of obstruccions. Where radio transmission is within the official UK medical and biological telemetry band (102.2-102.4 MHz), power must be limited to give a maximum effective range of about $200 \mathrm{~m}$. Where a greater range is required, it is necessary to use a frequency in another band within which higher transmission power is permitted, but it may be difficult to obtain a licence to operate in Britain in this way.

An idea of the likely size of the transmitter for any particular project may be gained from the examples illustrated in Fig. 2. The small transmitter manufactured by Devices Instruments Ltd for electrocardiograph (ECG) transmission (Fig. 2a) measures about $20 \times 20 \times 5 \mathrm{~mm}$. This includes the power pack. It has a range of about Io $m$ and a battery life of $\mathrm{I} 2 \mathrm{~h}$.
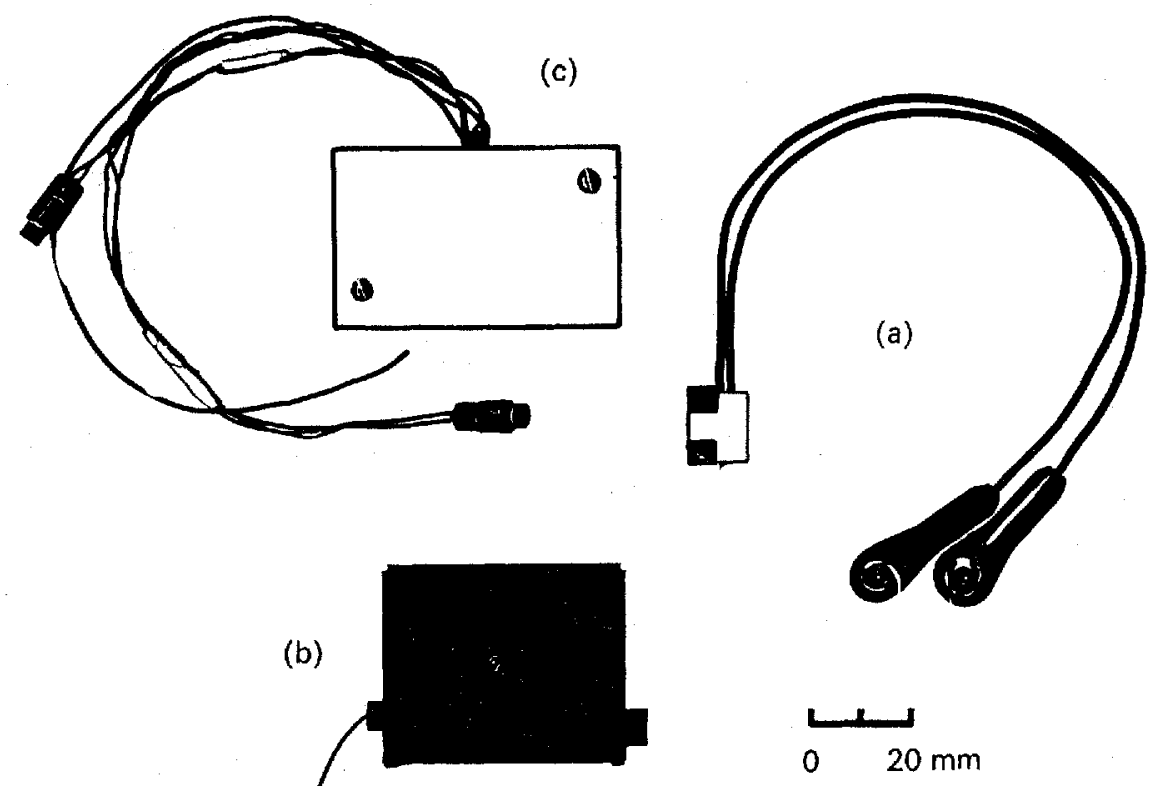

Fig. 2. Typical harness-borne radio-telemetry transmitters of biological information: (a) electrocardiac activity (ECG); (b) temperature; and (c) heat fow.

Fig. 2b shows a temperature transmitter built and used at Babraham. The wires from an implanted thermistor temperature sensor pass through the skin and connect to the transmitter held in a harness on the back of an animal. This device is usually set to transmit thermal information with an accuracy of $0 . I^{\circ}$ within a temperature range extending from $38.5^{\circ}$ to $42.5^{\circ}$. The range is variable and the system can also be used to record skin temperature over a different and wider range, and with the same percentage accuracy. The transmitter measures $55 \times 50 \times 20 \mathrm{~mm}$. The contained battery has a life of $80 \mathrm{~h}$ continuous operation.

The third transmitter (Fig. 2c) has a high-gain d.c. input stage, and will accept the input from almost any transducer which emits a d.c. electrical signal. It was originally 
designed for use with a Hatfield-Turner heat flow disc to record the flow of heat through the skin, but can also be used to transmit information about slowly changing physiological factors such as strain, pressure, air velocity, temperature or light intensity. This transmitter measures $70 \times 45 \times 25 \mathrm{~mm}$, and has a separate power pack of about the same size; this provides power for $120 \mathrm{~h}$ of continuous transmission.

If necessary, the electronic circuitry in all three transmitter units illustrated in Fig. I could be made much smaller by the use of modern components and techniques, but the cost would be considerable. Furthermore, since there have been no comparable improvements in the power:volume ratio of batteries (and these constitute approximately $50 \%$ of the total volume of most systems), the advantage of costly miniaturization is much reduced.

These transmitters are representative of a whole range of commercially available or readily constructable single-channel systems. The basic differences between transmitters depend on whether they are to be totally embedded, or carried externally, and whether they are to carry rapidly fluctuating (a.c.) or slowly fluctuating (d.c.) signals. Generally speaking, the small implantable types and the smaller of the harness-borne types are suitable for a.c. signals such as those derived from sensors of the electrical activities of the heart (ECG), muscles and nerves. Larger devices are necessary for the transmission of stable, long-term d.c. signals (e.g. of pressure or temperature information).

\section{Multi-channel systems}

In all physiological studies, the measurement of a single quantity is of limited interest. The relation of this quantity to concurrent events compels the measurement of other physiological functions. This is as true in the field as in the laboratory, and has stimulated the production of multi-channel systems of radio transmission. Separate transmitters for each quantity are generally unacceptable, both because of the size and weight of a multi-transmitter pack, and because a frequency band broad enough to permit adequate separation of the different transmitted signals may not be available. (Of course, these objections do not apply to the multiple implantation of radio pills, which transmit such a weak signal that the law relating to the transmission of radio signals is hardly applicable.)

The simplest multi-channel systems employ a single transmitter, into which the signals from several transducers can be fed sequentially by time-division-multiplexing, whereby a mechanical or electronic switching mechanism connects several transducers sequentially to the common transmitter (Fig. 3). Such multiplex systems require a means by which the recording device at the receiving station can be kept in step with the transmitter, and by which the different items of transmitter information can be identified. This is achieved by the transmission of a synchronizing pulse at the start of each cycle of transmission.

The sequential transmission of several items of information on a single carrier channel (time-division-multiplexing) is a slow process and permits the examination of each input signal only briefly and at a maximum rate of, say, one sample/s. For 

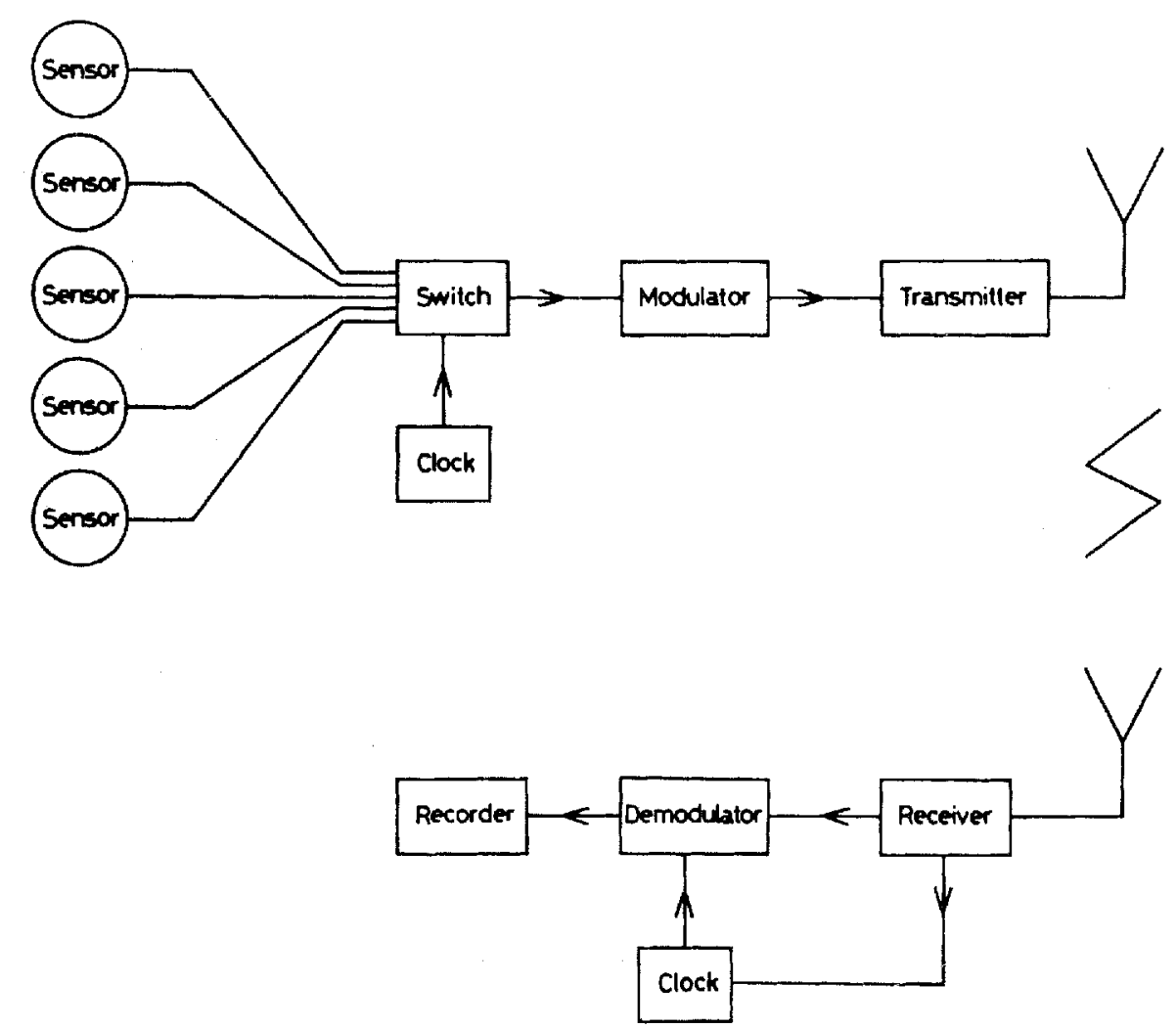

Fig. 3. A schematic representation of a multi-channel radio-telemetric system for transmitting and recording several different physiological measurements.

the transmission of several pieces of information at more frequent intervals, other multiplex systems which involve frequency-modulated oscillators or high-speed, digital-pulse techniques are available. Within the frequency widths available in the medical and biological waveband, it is possible to send about six channels of data independently, with responses from d.c. to about $50 \mathrm{~Hz}$ in each channel.

When the transmission of the higher audio frequencies is required, as for example, in studies of the electrical events in single nerve cells or the output of a Geiger-Müller tube, the use of individual transmission channels is the only possible method.

Multi-channel systems pose more problems than the equivalent number of singlechannel transmitters. These problems are largely related to the method of multiplexing. The synchronizing pulses of time-division-multiplex systems may be lost through electrical interference or a temporary fade of the signal, and the identity of the channels is lost. This problem becomes more acute as the number of channels is increased. Multiplex systems also require that all transducers have a common output format to match the single input stage. Simple calibration methods in which the transducer, transmitter, receiver and recorder are all calibrated together as a single unit thus become impossible, and each transducer must be adjusted to a standard 
output level. Despite these technical problems, a two-to-four-channel system for the monitoring of slowly changing input signals can be operated satisfactorily, particularly when the different pieces of transmitted information are qualitatively very similar, for example, skin temperature at four different positions.

Other forms of multiplexing also have their difficulties and, at present, there seems to be no entirely satisfactory system available for the maintained transmission, over a period of some days, of several d.c. signals. However, a number of multi-channel systems do exist for a.c. signals and for signals which indicate on-off information.

For the future, digital pulse systems are the most promising, and given the necessary finance for development, there is no doubt that a stable and efficient sixchannel transmitter could be built.

Many variations on the basic theme are possible. For example, the output of a short-range implanted transmitter can be received by a harness-borne unit and retransmitted over a much greater distance. Battery life can be extended by means of time switches or by a radio command on-off signal. In the instance of implanted transmitters, the power may be switched on by an external magnet.

Although radio-transmitted control of animal-borne instruments is not strictly within the scope of this discussion, it cannot be totally ignored. A radio receiver on an animal can receive and relay commands to mechanisms which, for example, operate syringes to inject fluids into an animal or withdraw fluids from the animal. Radio can also be used to switch on animal-borne recorders, and to operate release mechanisms by which these can be jettisoned from an unrestrained animal at a time and place chosen by an operator, for subsequent collection.

\section{Sensor-transducers of physiological variables}

Any physiological measurement which is electrical in nature (i.e. a biopotential), or which can be transduced into an electrical signal, can be caused to modulate a radio carrier wave which can be received, processed, displayed and recorded at a distance from the animal. Miniaturized and chronically implantable sensor-transducer devices are not exclusive to radio-telemetry, but the use of radio-telemetry has been attendant upon the development of such devices as well as upon the development of suitable radio systems.

The measurements that are now quite frequently sensed and transmitted by radio are: temperature, pressure, movement, fluid flow, heat flow, $\mathrm{pH}$ and biopotentials.

Temperature. Sensors for implanted transmitters usually consist of a temperaturecontrolled oscillator within the capsule (i.e. the sensor is part of the transmitter). For longer-range harness-mounted transmitters the temperature sensor is usually a suitably coated and embedded thermistor. The signal passes via wires through the skin to the transmitter and controls the frequency or mark:space ratio of an oscillator.

Pressure. This is mostly sensed and transduced into an electrical signal by the displacement of a strain gauge in a Wheatstone bridge circuit. This can be made contained in a small distortable capsule and implanted within a blood vessel or elsewhere in the body. Alternatively, in the instance of blood pressure, a cannula can be 
connected to an external strain gauge assembly which feeds a signal into a harnessborne transmitter. Bladder, alimentary tract and thoracic pressures can be similarly sensed and transmitted.

Movement. Movements of the whole animal, as in exercise, or in separate distinct structures, such as the rumen, and relating to separate functions, such as mastication, can be detected by a variety of resistance and inductance strain gauges suitably protected and anchored on or within the body, e.g. round the intestine or between two points on the rumen wall.

Fluid flow. This can be detected by electromagnetic and ultrasonic Döppler shift flowmeter systems which can be implanted round a vessel.

$p H$. The $\mathrm{pH}$ of body fluids, the stomach contents for example, can be monitored by an implanted miniature glass electrode.

Biopotentials. These do not need to be transduced. They are sensed by suitably placed or implanted electrodes linked to a pre-amplifier through a capacitance. ECG, electromyograph (EMG) and electroencephalograph (EEG) are sensed and fed to a transmitter in this way.

The principal technical difficulties experienced with implanted sensor-transducer devices are threefold.

(a) Encapsulation of the implant with fibrous tissue may interfere with the responsiveness of the sensor. For some measurements, such as movement, where the information is qualitative rather than quantitative, this may not be serious but for others, such as temperature and pressure, the prior calibration may become progressively less valid as the fibrous tissue accumulates. Thrombus formation on intravascular devices can cause an embolism, but pre-treatment of the implant with a surface smoothing agent, such as colloidal graphite, largely prevents this hazard.

(b) Recalibration of a sensor-transducer after implantation is virtually impossible, and this can be a serious handicap. However, the stability of the calibration of an implanted sensor can often be checked by comparing the record with that obtained from another freshly implanted sensor.

(c) The eventual fracture of wires leading from an implanted sensor is difficult to prevent, but it can sometimes be delayed by minimising the flexion to which the wires may be subjected as they pass through any two structures which move relative to each other, such as muscle and skin. Wires emerging from deep tissues should be lead subcutaneously for a distance before they are passed through the skin. Where the resistance of the connecting wires is not very important, multistrand cable can be used, since contact is maintained until the last strand breaks.

\section{Concluding comments}

There are three principal circumstances in which radio-telemetry offers a solution to basic problems: ( $I$ ) in field studies where an animal is unrestrained except within the range of satisfactory radio reception; (2) in the laboratory when the animal cannot be firmly restrained and when wired connections to recorders present problems; and (3) in the laboratory when physiological measurements are to be monitored over long periods without an observer in constant attendance. 
Although it is at the end of its second decade of development, radio-telemetry remains a largely unfulfilled hope. The high cost of development of some systems, particularly of those for recording several biological functions concurrently in a number of animals, has been barely repaid by the rather small volume of reliable and revealing results that has emerged. Good records showing several physiological measurements over a long period are still difficult to obtain, and it is the experience of most users of radio-telemetric systems that as much time, or more, is spent solving technical problems relating to the transducers or the transmitters as on the collection of worthwhile results. However, the promise of a means to study biological functions under more natural conditions than those of the buffered environment of the laboratory is too important to be ignored, and further developmental work merits support and encouragement.

At the moment, however, those who wish to embark on radio-telemetry would be well advised to appraise the situation before they start. It must be remembered that the radio carrier wave is only a convenient substitute for a wired connection between the animal and the recording system, but it greatly complicates this link. Before electing to use radio-telemetry and selecting a system, the following points must be specified: the necessary accuracy of the recorded data, the distance over which the data are to be transmitted, the acceptable battery-life of the transmitter, the biological measurements which must be recorded concurrently, whether the data records should be continuous or intermittent, the permissible dimensions and weight of the transmitter and power pack, and whether the transmitter assembly can be harness-borne. One then needs the help of someone competent to select, modify or design a system and to attend to the inevitable initial technical problems. 